\title{
Análise do impacto orçamentário cau- sado pela implantação de assinatura digital no Hospital das Clínicas da Faculdade de Medicina de Ribeirão Preto da Universidade de São Paulo
}

\section{Budget impact analysis caused by the implantation of digital signature at Clinics Hospital of Ribeirão Preto Medical School of University of São Paulo}

Felipe Carvalho Pellison ${ }^{1}$, Alexandre Carneiro², Eva Namburete ${ }^{3}$, Fabio Sant'Ana ${ }^{4}$, Lívia Roveri ${ }^{5}$, Marcos Roberto Pereira ${ }^{6}$, Tabata Tavares ${ }^{7}$, Antônio Pazin-Filho ${ }^{8}$

\begin{abstract}
RESUMO
Com o processo de inovação tecnológica, avanço de redes e softwares, surgem novas formas de catalogação de dados e documentos. No setor de saúde isso corrobora com a melhora do serviço prestado, já que o aumento da demanda requer segurança, rapidez e economia. Este trabalho foi realizado no Hospital das Clínicas da Faculdade de Medicina de Ribeirão Preto USP (HCFMRP USP), um hospital terciário que atua na assistência, ensino e pesquisa. Este trabalho tem como objetivo analisar o impacto orçamentário da aquisição e renovação do certificado digital pelo HCFMRP USP com uma análise direta do custo de impressão e aquisição de papel. Para o cálculo do tempo de retorno financeiro utilizou-se o Payback descontado. Verificou-se que o valor investido na aquisição do registro digital será totalmente revertido em aproximadamente menos de um ano, concluindo-se que esta migração é vantajosa em termos econômicos. A economia alcançada com este recurso proporcionará maior capacidade de investimento do hospital em outras áreas.
\end{abstract}

Palavras-chave: Custos e Análise de Custo; Economia Hospitalar; Registros Eletrônicos de Saúde.

1. Informata Biomédico; Mestrando em Gestão de Organizações de Saúde na Faculdade de Medicina de Ribeirão Preto da Universidade de São Paulo (FMRP-USP).

2. Farmacêutico; Mestrando em Gestão de Organizações de Saúde na FMRP-USP.

3. Médica; Residente na FMRP-USP.

4. Analista de Sistemas; Mestrando em Gestão de Organizações de Saúde na FMRP-USP.

5. Arquiteta; Mestrando em Gestão de Organizações de Saúde na FMRP-USP.

6. Bibliotecário; Mestrando em Gestão de Organizações de Saúde na FMRP-USP.

7. Médica; Mestrando em Gestão de Organizações de Saúde na FMRP-USP.

8. Médico; Professor Associado da FMRP-USP na área de Emergências Clínicas - Ribeirão Preto (SP), Brasil.
Correspondência:

Felipe Carvalho Pellison fcpellison@gmail.com

Seção Técnica de Informática Faculdade de Medicina de Ribeirão Preto - USP

Artigo recebido em 16/06/2014 Aprovado para publicação em 14/08/2014 


\begin{abstract}
With the process of technological innovation, networking, and software advances, new forms emerge cataloging data and documents. In the health sector it corroborates the improvement of service, as rising demand requires security, speed and economy. This work was performed at Clinics Hospital of Ribeirão Preto Medical School (HCFMRP) of University of São Paulo, a tertiary care hospital that operates in care, teaching and research. This work objective is to analyze the budgetary impact of the acquisition and renewal of digital certificates by HCFMRP with a direct analysis of the cost of printing and paper procurement. To calculate the payback time we used the Discounted Payback. It was verified that the amount invested in the acquisition of the digital record will be totally reversed in less than a year, therefore concluding that this migration is advantageous in economic terms. The savings achieved with this feature will provide greater ability to invest in other areas of the hospital.
\end{abstract}

Keywords: Costs and Cost Analysis; Economics, Hospital; Electronic Health Records.

\section{Introdução}

Atualmente passamos por um processo de informatização, o constante avanço das Tecnologias de Informação e Comunicação (TICs) associada ao aumento de infraestrutura como redes interconectadas e softwares com capacidades de interação, vem contribuindo para o desenvolvimento de novas formas de documentação e catalogação de informações em bancos de dados seguros e digitalizados.

A inserção das TICs em hospitais gera mudanças no cenário hospitalar atual, o que tem surgido como um grande desafio para instituições de saúde. É necessário acompanhar os avanços para atender a vasta demanda de serviços de maneira mais rápida, segura, econômica e prática.

Além das inovações tecnológicas no campo médico, observadas nas mudanças de procedimentos de rotina, novas técnicas cirúrgicas e medicamentos, também se mostram com grandes necessidades de inovações na gestão hospitalar. ${ }^{1}$ Goldstein ${ }^{2}$ enfatiza que os hospitais são instituições de saúde com certo grau de complexidade, onde todos os dias surgem novos desafios para a sua gestão.

Nesse contexto, torna-se imprescindível a implantação de novas tecnologias dentro dessas instituições, visando o êxito na gestão de seus respectivos setores e trazendo consequentemente, mudanças satisfatórias e que possam garantir a qualidade do atendimento.

O Hospital de Clinicas da Faculdade de Medicina de Ribeirão Preto da Universidade de São Paulo (HCFMRP-USP) atua na assistência, ensino e pesquisa desde sua inauguração em 1956, segundo o Relatório de Gestão 2003 a 2010. Atualmente conta com uma área construída de $123.138,77 \mathrm{~m}^{2}$, contem- pla 1.099 leitos e atende as mais variadas especialidades. Segundo o seu regulamento*, o HCFMRP é vinculado à Secretaria de Governo para fins administrativos e associa-se à USP para fins de ensino, pesquisa e prestação de serviços médico-hospitalares à comunidade.

Na composição do HCFMRP-USP estão: ambulatórios, unidades de internação, laboratórios, administração, unidades de apoio, entre outras. O hospital conta com uma estrutura capaz de proporcionar treinamento e ensino para profissionais e estudantes em cursos de graduação, pós-graduação e aperfeiçoamento. $^{3}$

Dentre as diversas áreas que compõem o HCFMRP este trabalho terá atuação no Centro de Informações e Análise (CIA) do HCFMRP-USP, setor do hospital responsável pela gestão e elaboração de tecnologias. O setor, dentre outras atribuições, busca criar, inserir e aprimorar tecnologias utilizadas na rotina do hospital.

Recentemente o Prontuário Eletrônico do Paciente foi desenvolvido pelo CIA para o hospital, o que permitiu a adoção dos prontuários de pacientes totalmente eletrônicos, da mesma forma como é realizado em papel. O PEP é o sistema com mais acesso do complexo.

Com a evolução da tecnologia, especialmente da Internet, o compartilhamento das informações de saúde tornou-se mais simples e possível, naturalmente o PEP, antes de uso exclusivo e interno da instituição de saúde, evoluiu para o conceito de um Registro Eletrônico de Saúde (RES). O RES possui em seu núcleo conceitual o compartilhamento de informações

\footnotetext{
* Disponível em http://www.hcrp.fmrp.usp.br/sitehc/arqs/pdf/ REGULAMENTO_VIGENTE_II.pdf acessado em 09/10/2014.
} 
sobre a saúde de um ou mais indivíduos, inter e multiinstituição, dentro de uma região (município, estado ou país), ou ainda, entre um grupo de hospitais.

Sendo assim, o documento com as informações do paciente será totalmente digitalizado. Segundo Moacyr ${ }^{4}$, pode-se entender por documento a "coisa representativa de um fato". Dessa forma, outro autor relata que ${ }^{5}$, "documento eletrônico pode ser entendido como a representação de um fato concretizado por meio de um computador e armazenado em formato específico (organização singular de bits e bytes), capaz de ser traduzido ou apreendido pelos sentidos mediante o emprego de programa (software) apropriado".

Para a total digitalização dos documentos e informações dos pacientes em um hospital, é necessária a utilização da assinatura digital, que garante, digitalmente, a autoria de qualquer informação acrescentada ou alterada. Com a assinatura digital fica registrado quem é o usuário, onde esse usuário entrou, o que acessou e o que acrescentou, sendo que esse registro é único e exclusivo àquele usuário específico. Existe também a garantia de que a mensagem não foi alterada no processo de transmissão, o que garante além da autenticação de origem, a integridade da mensagem ${ }^{6}$.

Para que a assinatura digital tenha validade legal, no Brasil, as Instituições de Saúde devem obter uma certificação, que só é possível se a instituição seguir as orientações do Manual de Certificação para Sistemas de Registro Eletrônico em Saúde.

\section{Conceito e importância do certificado digital}

O Certificado Digital é uma assinatura digital com validade jurídica, ou seja, é um arquivo de computador que identifica uma pessoa física ou jurídica no mundo digital, que garante proteção às transações eletrônicas e outros serviços via internet, permitindo que pessoas e empresas se identifiquem e assinem digitalmente de qualquer lugar do mundo com mais segurança e agilidade. Segundo o Instituto Nacional de Tecnologia da Informação (ITI), o "certificado digital é um documento eletrônico que contém o nome, um número público exclusivo denominado chave pública e muitos outros dados que mostram quem somos para as pessoas e para os sistemas de informação. A chave pública serve para validar uma assinatura realizada em documentos eletrônicos"7.

Um Certificado Digital normalmente apresenta as seguintes informações ${ }^{8}$ :

- Nome da pessoa ou entidade a ser associada à chave pública
- Período de validade do certificado chave pública

- Nome e assinatura da entidade que assinou o certificado

- Número de série.

O certificado digital tem diversas finalidades, tais como garantir a comunicação segura entre dois sistemas e criptografar o acesso a diversos websites.

São utilizadas diversas técnicas computacionais para garantir que o documento eletrônico assinado por um certificado digital seja totalmente seguro. Podese comparar o certificado digital com a sua impressão digital, já que cada documento assinado possui um identificador único e até mesmo uma pequena alteração no documento, como a simples inserção de um espaço em branco, invalida totalmente o documento, garantindo assim a sua integridade.

O certificado Digital traz varios benefícios tais como: Economia de tempo, validade jurídica nos documentos eletrônicos, desburocratiza processos, pois não necessita de reconhecimento de firma, economia ambiental e ganho financeiro uma vez que deixa de ser impressa uma enorme quantidade de papel. ${ }^{9}$

O Manual de Certificação para Sistemas de Registro Eletrônico em Saúde ${ }^{10}$, desenvolvido pelo Conselho Federal de Medicina em conjunto com a Sociedade Brasileira de Informática em Saúde, tem por objetivo fornecer requisitos para o desenvolvimento de Sistema de Registro Eletrônico de Saúde (S-RES), para que estejam em conformidade com as boas práticas da informática em saúde. Para tanto, dentre muitas outras recomendações, o documento estabelece dois "Níveis de Garantia de Segurança", NGS1 e NGS210, que devem ser alcançados a partir de suas recomendações ${ }^{11}$. No que tange ao uso de registros eletrônicos em detrimento do papel, a extinção do segundo só pode ocorrer quando o primeiro alcançar o NGS2* . Neste estágio é exigida a operação através do uso de certificado digital ${ }^{7}$.

Uma das grandes vantagens da utilização do registro eletrônico aliado ao PEP estudados pelo HCRP é o fato de ser possível a eliminação de impressão de documentos médicos em papel. Outro fato importante dentro dessa discussão é que o prontuário em papel apresenta diversas limitações tais como: impossibilidade de acesso simultâneo, as informações em papel

\footnotetext{
* As exigências de cada um dos níveis podem ser encontradas em: http://portal2.tcu.gov.br/portal/pls/portal/docs/2061290.PDF
} 
não podem disparar lembrete ou alertas aos profissionais, para estudos cientificos as informações contidas no prontuário em papel precisam ser transcritas, fatos que pode predispor erros ou omissões de informações. Além disso, apresenta uma série de desvantagens, tais como: elegibilidade das informações contidas, redundância dos dados, fragilidade do papel, por acarretar perda de informações com o passar do tempo, necessidade de maior espaço físico para armazenamento e organização de grande volume de dados. ${ }^{12}$

A eliminação de papéis permite a diminuição de espaços para arquivamentos, maior preservação de documentos e garantia de integridade dos documentos que, sob o modelo tradicional, por serem manipulados com bastante frequência, podem sofrer avarias ou até mesmo serem desordenados, inviabilizando a sua leitura. Além disso, a redução de papel em hospitais pode ter consideráveis impactos econômicos.

A busca constante por redução de custos e aumento de eficiência é uma pratica de qualquer empresa, contudo no âmbito da administração de hospitais públicos, tal situação torna-se uma questão de sobrevivência.

Portanto faz-se necessário um posicionamento estratégico das organizações da área da saúde para a manutenção de seus recursos financeiros, bem como a escolha de ferramentas de Tecnologia de informação capazes de trazer benefícios para as organizações de saúde.

Vários estudos ao redor do mundo têm demonstrado o impacto positivo sobre a Saúde que a implementação de um PEP/RES pode trazer tanto para os profissionais de saúde, como para os pacientes, gestores e toda a equipe envolvida na Atenção à Saúde.

Este estudo se justifica pela possibilidade de substituição de um procedimento existente (impressão em papel) por outro, aparentemente, com melhor custo benefício para o hospital, que permitirá melhor proveito do investimento, podendo utilizar mais coerentemente os recursos financeiros que poderão ser empregados em outras necessidades.

\section{Objetivo}

O presente trabalho tem como objetivo a projeção do retorno financeiro em um período de 6 anos, considerando a aquisição e renovação das assinatura eletrônica pelo HCFMRP-USP e os custos envolvidos no processo de impressão em papel após a implentação dos certificados.

\section{Metodologia}

\section{Análise de custo de aquisição de certificado digital}

Serão analisados os efeitos do impacto orçamentário da aquisição de 4300 certificados digitais, de acordo com licitação realizada pelo HCFMRP em 2014, em detrimento da impressão de vários documentos comuns ao dia-a-dia hospitalar. Tais certificados possuem validade de 3 anos e, após este período, há necessidade de renovação.

A análise considera a infraestrutura de Tecnologia da Informação (TI) existente no hospital como resultado de investimentos prévios. Portanto, não é escopo do projeto levar em consideração quaisquer custos de datacenter, hardware, recursos humanos, energia e manutenção de toda infraestrutura.

\section{Análise de custo de impressão}

Será realizada uma análise de custo direta em relação ao ano de 2013 levando-se em consideração os seguintes impressos: evolução e observação clínica, protocolo de assistência de enfermagem, laudos de exames e exames laboratoriais. Este cálculo considerará o custo da aquisição de papéis pelo hospital e impressão, que atualmente é realizado através de regime comodato, onde existe um contrato de "aluguel" de impressoras e suprimentos sob demanda

Não é escopo de este estudo analisar custos referentes ao armazenamento, digitalização, transporte e recursos humanos relacionados ao processo de manutenção destes impressos. Também não será considerado para efeito deste estudo o impresso de prescrições médica dada a alta variabilidade no número de impressões.

\section{Contratação e demissão de funcionários}

Será estabelecida uma média de contratação e demissão de funcionários no hospital (considerando os anos 2012 e 2013, por instituição - HC e FAEPA), baseada em informações obtidas junto ao CIA. Essas informações são necessárias para que se possa ter ciência do número de aquisições e renovações de certificados digitais ao longo do período de estudo.

\section{Método Payback}

Quando existe intenção de fazer um investimento, a primeira observação que precisa ser feita é referente ao retorno do investimento. Quanto tempo será necessário para que se recupere investimento? Essa 
resposta, em conjunto com diversos outros fatores inerentes, tem impacto direto na tomada de decisão do gestor responsável.

O método sugerido para este estudo é o Payback Descontado ${ }^{13}$ por levar em consideração a taxa de desconto sofrida por cada fluxo de caixa e apresentar uma estimativa de quando o valor de investimento (fluxo negativo) se anulará com o valor retornado pelo investimento (fluxo positivo). ${ }^{14} \mathrm{Tal}$ taxa de desconto deve ser levada em consideração pois qualquer montante atual não terá o mesmo valor no futuro, ou seja, será depreciado, principalmente, pela inflação. A expressão genérica ${ }^{15}$ utilizada para o cálculo do Payback Descontado pode ser representada da seguinte forma:

$$
\mathrm{FCC}(\mathrm{t})=-\mathrm{I}+\sum_{\mathrm{j}=1}^{\mathrm{t}} \frac{\left(\mathrm{R}_{\mathrm{j}}-C_{j}\right)}{(1+i)^{j}} ; \quad 1 \leq \mathrm{t} \leq \mathrm{n}
$$

Onde:

$\boldsymbol{F C C}(\boldsymbol{t})$ é o valor presente do capital, ou seja, o fluxo de caixa descontado para o valor presente cumulativo até o instante t;

I é o investimento inicial (em módulo), ou seja, -I é o valor algébrico do investimento, localizado no instante 0 (início do primeiro período);

$\boldsymbol{R} \boldsymbol{j}$ é a receita proveniente do ano $j$;

$\boldsymbol{C} \boldsymbol{j}$ é o custo proveniente do ano $j$; $\boldsymbol{i}$ é a taxa de juros empregada (neste caso a depreciação causada pela inflação).

$\boldsymbol{j}$ é o índice genérico que representa os períodos (neste caso os anos), por exemplo, $t=0$ momento do investimento, $t=1$ primeiro ano após o investimento, $\boldsymbol{t}=\mathbf{n} n$-ésimo ano após o investimento;

Atinge-se o valor de Payback Descontado quando $F C C(t)=0$, onde $t$ será o ano após o investimento. Conforme ressaltado ${ }^{13}$, se ocorrer $F C C(t)<0$ em $j-1$, realizamos a interpolação $(t i)$ para determinar o momento fracionado, isto é: $t<t i<t+1$.

Com o auxílio do Centro de Informações e Análise, foram levantados os dados de consumo de impressos do Sistema de Gestão de Materiais para o ano de 2013 que compõem a Tabela 1.

Para efeito dos cálculos, levamos em consideração que ainda haverá uma impressão residual de $5 \%$ deste montante de impressos ocasionados por eventuais solicitações de paciente, processos judiciais e demais motivos que justifiquem a impressão. Portanto a impressão não será abolida totalmente e seu custo não será revertido integralmente como fluxo positivo de caixa.

De acordo com a licitação realizada pelo Hospital, temos os seguintes custos para o certificado digital (Tabela 2).

Tabela 1: Consumo de impressos para o ano de 2013 (Sistema de Gestão de Materiais)

Impresso

Sistematização da Assistência de Enfermagem

Observações e Evoluções Clínicas

Impressão de resultados de exames (LIS + Laudos)

Papel Sulfite para impressão de exames

\section{Custo}

$\mathrm{R} \$ 37.378,38$

$R \$ 142.561,85$

$\mathrm{R} \$ 61.920,00$

$\mathrm{R} \$ 28.800,00$

Tabela 2: Quantidades e custos para a aquisição e renovação dos Certificados Digitais.

Número total de Certificados Comprados

Custo total (em R\$) dos Certificados comprados

Custo (em R\$) de cada Certificado

Número de Certificados Utilizados

Custo (em R\$) dos Certificados Utilizados

Custo de renovação de 1 certificado
5036

$R \$ 247.006,68$

$R \$ 49,05$

4300

$\mathrm{R} \$ 210.907,21$

$\mathrm{R} \$ 49,05$

Fonte: Elaboração própria. 
Para simularmos a rotatividade de funcionários que ocorre no Hospital, foram levantados através do Sistema de Recursos Humanos os números de demissões e contratações tanto via FAEPA quanto via HCRP para os anos de 2012 e 2013, apresentados na Tabela 3.

A partir deste levantamento realizamos uma média para estimarmos os valores de contratação e demissão anual de cada instituição, apresentado na tabela 4.

Tabela 3: Contratações e demissões via FAEPA e HC (Sistema de Recursos Humanos)

\begin{tabular}{lcc} 
& \multicolumn{2}{c}{ Ano } \\
\cline { 2 - 3 } & $\mathbf{2 0 1 2}$ & $\mathbf{2 0 1 3}$ \\
\hline Contratações FAEPA & 184 & 197 \\
Contratações HC & 279 & 414 \\
Demissões HC & 201 & 361 \\
Demissões FAEPA & 192 & 321 \\
\hline
\end{tabular}

Fonte: Elaboração própria.
Tabela 4: Estimativa de contratação e demissão anual por instituição.

$\begin{array}{lc}\text { Estimativa Anual de Demissões FAEPA } & 257 \\ \text { Estimativa Anual de Demissões HC } & 281 \\ \text { Estimativa Anual de Contratações FAEPA } & 191 \\ \text { Estimativa Anual de Contratações HC } & 347\end{array}$

Fonte: Elaboração própria.

Para o cálculo do Payback descontado a taxa de desconto utilizada será o valor sugerido ${ }^{13}$ que é de $8 \%$ ao ano.

A partir de tais valores, foram feitas as estimativas de fluxo de caixa para 6 anos futuros, onde a aquisição e início do uso dos certificados digitais se dá no começo da iteração 0 , simulado como 2015. Tal fluxo de caixa detalhado é apresentado na Tabela 5.

$\mathrm{Na}$ Tabela 6 trazemos os valores calculados de fluxo de caixa cumulativo descontado (que leva em consideração a taxa de desconto, ou seja, a inflação) bem como o fluxo de caixa cumulativo simples (que não leva em consideração a taxa de desconto).

Tabela 5: Fluxo de caixa estimado do ano 2015 a 2014

\begin{tabular}{|c|c|c|c|c|c|c|}
\hline \multirow[b]{2}{*}{ Ano } & \multicolumn{6}{|c|}{ Cálculo de fluxo de caixa } \\
\hline & 2015 & 2016 & 2017 & 2018 & 2019 & 2020 \\
\hline Iteração (j) & 0 & 1 & 2 & 3 & 4 & 5 \\
\hline \multicolumn{7}{|l|}{ Fluxo (em R\$) } \\
\hline $\begin{array}{l}\text { Sistematização da Assistência de } \\
\text { Enfermagem }\end{array}$ & 35509,46 & 35509,46 & 35509,46 & 35509,46 & 35509,46 & 35509,46 \\
\hline Observações e Evoluções Clínicas & 135433,76 & 135433,76 & 135433,76 & 135433,76 & 135433,76 & 135433,76 \\
\hline $\begin{array}{l}\text { Impressão de resultados de } \\
\text { exames (LIS + Laudos) }\end{array}$ & 58824,00 & 58824,00 & 58824,00 & 58824,00 & 58824,00 & 58824,00 \\
\hline $\begin{array}{l}\text { Papel Sulfite para impressão } \\
\text { de exames }\end{array}$ & 27360,00 & 27360,00 & 27360,00 & 27360,00 & 27360,00 & 27360,00 \\
\hline Compra Inicial de Certificados & $-210907,21$ & 0,00 & 0,00 & 0,00 & 0,00 & 0,00 \\
\hline Renovação de Certificados & 0,00 & 0,00 & 0,00 & $-158131,36$ & 0,00 & 0,00 \\
\hline $\begin{array}{l}\text { Custo de Certificados para } \\
\text { Recém-Contratados (FAEPA) }\end{array}$ & $-9368,20$ & $-9368,20$ & $-9368,20$ & $-9368,20$ & $-9368,20$ & $-9368,20$ \\
\hline $\begin{array}{l}\text { Custo de Certificados para } \\
\text { Recém-Contratados (HC) }\end{array}$ & $-17019,72$ & $-17019,72$ & $-17019,72$ & $-17019,72$ & $-17019,72$ & $-17019,72$ \\
\hline Total por ano & 19832,08 & 230739,29 & 230739,29 & 72607,93 & 230739,29 & 230739,29 \\
\hline
\end{tabular}


Tabela 6: Cálculo do payback descontado com o fluxo de caixa cumulativo descontado

\begin{tabular}{|c|c|c|c|c|c|c|}
\hline \multirow[b]{2}{*}{ Ano } & \multicolumn{6}{|c|}{ Payback Descontado } \\
\hline & 2015 & 2016 & 2017 & 2018 & 2019 & 2020 \\
\hline Iteração (j) & 0 & 1 & 2 & 3 & 4 & 5 \\
\hline Inflação & 0,0800 & 0,0800 & 0,0800 & 0,0800 & 0,0800 & 0,0800 \\
\hline Fluxo de Caixa Pontual (em R\$) & 19832,08 & 230739,29 & 230739,29 & 72607,93 & 230739,29 & 230739,29 \\
\hline $\begin{array}{l}\text { Fluxo de Caixa Cumulativo Simples } \\
\text { (em } R \$)\end{array}$ & 19832,08 & 250571,37 & 481310,67 & 553918,60 & 784657,89 & 1015397,18 \\
\hline Valor Presente Descontado (em R\$) & 19832,08 & 213647,49 & 197821,75 & 57638,52 & 169600,27 & 157037,29 \\
\hline $\begin{array}{l}\text { Fluxo de Caixa Cumulativo } \\
\text { Descontado (em R\$) }\end{array}$ & 19832,08 & 233479,57 & 431301,33 & 488939,84 & 658540,11 & 815577,40 \\
\hline
\end{tabular}

Nota-se que o período de Payback calculado utilizando-se os dados levantados é extremamente baixo, em menos de 1 ano ocorre o retorno completo do investimento e ainda há um fluxo de caixa positivo de cerca de $\mathrm{R} \$ 20.000,00$.

Mesmo ao final do período de 6 anos, levando-se em consideração custos altos de renovação dos certificados digitais, estagnação no número de impressões e um cenário em que o Índice Geral de inflação não é dos mais otimistas dado o histórico recente, temos um fluxo cumulativo de caixa descontado de aproximadamente $\mathrm{R} \$ 815.000,00$ que pode ser verificado no Gráfico 1.

\section{Conclusão}

Baseado nas estimativas expostas conclui-se que o valor investido na aquisição do registro digital será totalmente revertido em menos de um ano.

Esses dados demonstram, de forma bastante otimista, as transformações em que se insere o sistema de saúde brasileiro, sendo inevitável a migração para o sistema digital. Podemos demonstrar que essa migração é vantajosa em termos econômicos e, com isso, enfatizar a necessidade de impulsionar o uso desse sistema em detrimento do uso de impressão.

Em longo prazo, a economia alcançada pela aquisição do registro digital, proporcionará maior capacidade de investimento do hospital em áreas de escassez de recursos. O planejamento poderá buscar relocar os recursos e com isso diminuir o distanciamento entre as qualidades e as deficiências das diferentes áreas de atuação do HCFMRP, o que está em perfeita consonância com uma de suas principais diretrizes, o constante aperfeiçoamento e melhoria contínua das atribuições e serviços prestados.

\section{Referencias}

1. Mota FRL. Prontuário Eletrônico do Paciente e o Processo de Competência Informacional. s.e. Florianópolis, 2006.

2. Goldstein MM. Health Information Technology and the Idea of Informed. IN: Journal of Law, Medicine \& Ethics. The effects of health information technology on the physician-patient relationship, PP. 27-35, spring 2010. 
3. Silva de Sá MF. Hospital das Clinicas da Faculdade de Medicina de Ribeirão Preto da Universidade de Ribeirão Preto Histórico HCRP. Medicina (Ribeirão Preto). 2002; 35:397-402.

4. Santos MA. Primeiras Linhas de Direito Processual Civil, vol. II, p. 386.

5. Castro AA. O documento eletrônico e a assinatura digital (Uma visão geral). Disponível em: <http://www.aldemario.adv.br/ doceleassdig.htm >. Acessado em: 02/10/2014.

6. CFM, 2012. Conselho Federal de Medicina; Sociedade Brasileira de Informática em Saúde. Cartilha sobre prontuario eletrônico: a certificação de sistemas de reistro eletrônico de saúde. Brasilia, 2012. Disponível em: <http://portal.cfm.org.br/ crmdigital/Cartilha_SBIS_CFM_Prontuario_Eletronico_fev_ 2012.pdf>. Acessado em: 24/09/2014.

7. BRASIL. Ministério da Saúde. Portaria 2073 de 31/08/2011. Brasilia, 2011. Disponível em: <http://bvsms.saude.gov.br/bvs/ saudelegis/gm/2011/prt2073_31_08_2011.htm> Acessado em: 24/09/2014.

8. Conselho Federal de Medicina; Sociedade Brasileira de Informática em Saúde. Manual de Certificação para Sistemas de Registro Eletrônico em Saúde . Brasilia, 2007. Disponível em: <http://portal2.tcu.gov.br/portal/p/s/portal/docs/ 2061290.PDF> Acessado em: 24/09/2014.
9. Marques EP, Oliveira GLB, Kiatake LGG, Carvalho Júnior MA, Silva ML, Bernal VB. Manual de Certificação para Sistemas de Registro Eletrônico em Saúde (E-RES), versão 4.1, Certificação 2013. Disponível em: <http://www.sbis.org.br/ certificacao/Manual_Certificacao_SBIS-CFM_2013_v41.pdf > Acessado em: 09/10/2014.

10. Brealey RA; Myers SC; Marcus AJ. Fundamentals of Corporate Finance, $3^{\underline{a}}$ ed. 2001.

11. Schaicoski JC. A utilização do ROI na análise de projetos de tecnologia da informação, 2002. - Universidade Federal de Santa Catarina, Florianópolis, 2002.

12. Bertolo LA. Payback. [20—?]. Disponível em: <http:// www.bertolo.pro.br/AdminFin/Anallnvest/PAY\%20BACK.pdf> Acessado em: 24/09/2014.

13. Sean DS. Budget Impact Analysis-Principles of Good Practice: Report of the ISPOR 2012 Budget Impact Analysis Good Practice II Task Force, 2012 - University of Washington, Seattle, Estados Unidos da América, 2014. 